\title{
Body Contouring Surgery Following Bariatric Surgery and Dietetically Induced Massive Weight Reduction: A Risk Analysis
}

\author{
S. de Kerviler • R. Hüsler • A. Banic • \\ M. A. Constantinescu
}

Received: 15 April 2008 / Accepted: 4 August 2008/Published online: 27 August 2008

(C) Springer Science + Business Media, LLC 2008

\begin{abstract}
Background This study analyzed the impact of weight reduction method, preoperative, and intraoperative variables on the outcome of reconstructive body contouring surgery following massive weight reduction.

Methods All patients presenting with a maximal BMI $\geq$ $35 \mathrm{~kg} / \mathrm{m}^{2}$ before weight reduction who underwent body contouring surgery of the trunk following massive weight loss (excess body mass index loss (EBMIL) $\geq 30 \%$ ) between January 2002 and June 2007 were retrospectively analyzed. Incomplete records or follow-up led to exclusion. Statistical analysis focused on weight reduction method and pre-, intra-, and postoperative risk factors. The outcome was compared to current literature results.

Results A total of 104 patients were included ( 87 female and 17 male; mean age 47.9 years). Massive weight reduction was achieved through bariatric surgery in 62 patients $(59.6 \%)$ and dietetically in 42 patients (40.4\%). Dietetically achieved excess body mass index loss (EBMIL) was $94.20 \%$ and in this cohort higher than surgically induced reduction EBMIL $80.80 \%(p<0.01)$. Bariatric surgery did not present increased risks for complications for the secondary body contouring procedures. The observed complications (26.9\%) were analyzed for risk factors. Total tissue resection weight was a significant risk factor $(p<0.05)$. Preoperative BMI had an impact on infections $(p<0.05)$. No impact on the postop-
\end{abstract}

None of the authors had a commercial interest in the subject of the study or financial benefit from the results.

S. de Kerviler $(\bowtie) \cdot$ R. Hüsler $\cdot$ A. Banic $\cdot$ M. A. Constantinescu Department of Plastic and Hand Surgery, Inselspital,

Bern University Hospital, University of Bern,

Freiburgstrasse,

3010 Bern, Switzerland

e-mail: sora.dekerviler@insel.ch erative outcome was detected in EBMIL, maximal BMI, smoking, hemoglobin, blood loss, body contouring technique or operation time. Corrective procedures were performed in 11 patients $(10.6 \%)$. The results were compared to recent data.

Conclusion Bariatric surgery does not increase risks for complications in subsequent body contouring procedures when compared to massive dietetic weight reduction.

Keywords Bariatric surgery · Body contouring . Morbid obesity . Complications

\section{Introduction}

Obesity is today considered as a 'disease' taking epidemic proportions, frequently associated with increased morbidity and mortality as well as economic health costs. About 300 million people around the world are obese (BMI>30). In the USA nearly two thirds of the population can be classified as overweight or obese [1], while in most European countries obesity rates tripled over the past two decades. The prevalence of obesity varies significantly among countries, with France and Switzerland showing the lowest obesity prevalence, and Slovenia, Croatia, and Greece the highest prevalence with similar distribution in men and women between $4-16 \%[2,3]$. Morbid obesity is defined as a BMI of over 40 or a BMI over 35 in combination with comorbidities [4]. Related comorbidities, like diabetes mellitus, arterial hypertension, sleep apnea syndrome, cardiovascular disease, hyperlipidemia, degenerative joint disease, and depression are known to reduce the quality of life and threaten the overall life expectancy of severely obese persons [5]. Bariatric surgery is an expanding field, given the significant negative impact of morbid 
obesity and the limited efficacy of dietetic therapy [6, 7]. Data from The American Society of Bariatric Surgeons show that the number of surgical procedures performed to induce weight loss has increased from 28,800 in 1999 to 171,000 in 2005 , with subsequent increasing number of patients who require secondary corrective procedures as surgical steps towards normal quality of life.

Patients frequently continue to suffer from stigmatizing sequels in the form of redundant skin and encumbering soft tissues, which interfere with exercise, proper fitting of clothes, and present not only an aesthetic burden but can be painful through mechanical friction and pose a hygienic problem associated with fungal infections and intertriginous dermatitis $[8,9]$. According to the American Society of Plastic Surgeons, over 68,000 body contouring procedures were performed for massive-weight-loss patients in 2005 [1]. The plastic and reconstructive surgeon must address these secondary deformities and choose the appropriate surgical technique and the optimal time point for surgery while assessing the operative risks for each individual. Most current investigations focus on the risk of secondary procedures in the post-bariatric surgical population and only few risk factors have been so far statistically outlined while others are still being controversially discussed [10-14].

To date it remains unclear whether the particular method of weight reduction (dietetic vs. bariatric) may additionally predispose for increased complication rates in the secondary reconstructive body contouring procedures. The introduction of excess body mass index loss (EBMIL) as an accurate measure tool for individual weight loss [15] independent of the therapeutic approach facilitates this comparison.

This study was designed to investigate and define risk factors for complications in reconstructive body contouring surgery following different methods of massive weight reduction. The results were further compared to the outcome reported in current studies $[10,11]$ and with previous data from our institution [13].

\section{Patients and Methods}

All patients undergoing reconstructive body contouring procedures following massive weight loss between January 2002 and June 2007 at our institution were analyzed retrospectively in this study. Inclusion criteria were a BMI of $\geq 35 \mathrm{~kg} / \mathrm{m}^{2}$ (BMI max.) before weight loss and a successful weight reduction as defined by an EBMIL $\geq 30 \%$ followed by a stable weight plateau maintained for at least 12 months prior to the body contouring procedure. Patients who were lost at follow-up or presented incomplete documentation were excluded.

Following data and variables were collected from the patients' medical records for statistical analysis: gender, age, maximal BMI before weight reduction (BMI max), BMI prior to body contouring surgery (BMI preop), excess body mass index loss, method of weight reduction (dietetic vs. bariatric surgery), nicotine consumption, hemoglobin levels, operative technique, intraoperative findings, blood loss, total operation time, and total tissue resection weight.

Outcome variables included conservatively treated minor complications (seromas, hematomas, local infections, small skin necrosis or wound dehiscence below $4 \mathrm{~cm}^{2}$ ), and major complications requiring surgical revision. Furthermore, tertiary corrective procedures for dog ears or scars were noted.

\section{Surgical Technique}

The reconstructive body contouring procedures were performed under general anesthesia, muscle relaxation and perioperative antibiotic coverage (1.5 g cefuroxime iv.). Abdominoplasty was defined as complete undermining of the skin and subcutaneous fat up to the xyphoid process with umbilical preservation according to the method described by Pitanguy et al. [16] and combinations thereof with median soft tissue resection. Rectus sheath diastases exceeding $4 \mathrm{~cm}$ were repaired by fascial plication. Incisional hernias $<2 \mathrm{~cm}$ were repaired primarily, larger defects with a mesh in an onlay or sub-fascial location. Wound closure was routinely performed with resorbable sutures in three layers: Scarpa fascia with 2-0 vicryl, subcuticular stitches with 3-0 monocryl followed by a 3-0 monocryl intracutaneous running suture. Blood transfusions were performed hemoglobin dependent intraoperatively and symptoms oriented in the postoperative phase. Circumferential skin excess of the trunk was corrected by belt lipectomy. Excess soft tissues of the thighs, arms, and breasts were resected separately depending on the individual patient need.

All patients received prophylactic respiratory physiotherapy, were mobilized within $12 \mathrm{~h}$ of surgery and received antithrombotic therapy until discharge from hospital. The drains were removed when drainage decreased to less than $20 \mathrm{ml}$ per day and drain. Patients undergoing abdominal wall plication or hernia repair were encouraged to wear a waist binder for 8 weeks.

Postoperative controls were scheduled routinely at 1,2 , 4 weeks, 3 and 6 to 12 months following reconstructive body contouring.

\section{Statistical Analysis}

The investigation included descriptive statistics of means with standard deviation and medians, depending on the distribution of the data. Differences in patient treatment characteristics between dietetic and bariatric surgery group were assessed by non-parametric tests (Mann-Whitney, 
Fishers-Exact, Kruskal-Wallis), which were used to compare means. Linear and multiple regression models (ANOVA, MANOVA) were applied in a second step to analyze risk factors for complication in the different treatment groups. SAS 9.1 (SAS Institute Inc., Cary, NC, USA) was used for all analyses. The level of significance was set at 0.05 throughout the study.

\section{Results}

A total of 112 patients met the first inclusion criterion of a BMI of $\geq 35 \mathrm{~kg} / \mathrm{m}^{2}$ (BMI max.) before weight loss, however only 109 patients met the harder second inclusion criteria of an EBMIL $\geq 30 \%$ followed by a stable weight plateau maintained for at least 12 months prior to the body contouring procedure. Five patients were excluded due to incomplete records. The remaining 104 patients undergoing reconstructive body contouring procedures were enrolled in the study. The mean age was 47.9 years, 17 patients were male, 87 patients were female. Median follow-up time was 11.1 months (5-28 months) excluding one lethal outcome due to pulmonary embolism. The cohort details are summarized in Table 1.

Weight reduction was achieved through various approaches of bariatric surgery in 62 patients $(59.6 \%)$ and by dietetic measures in 42 patients (40.4\%) (Fig. 1). The mean overall maximal BMI before weight reduction was $46.76 \mathrm{~kg} / \mathrm{m}^{2}$. Dietetic therapy started at a significantly lower median BMI of $40.4 \mathrm{~kg} / \mathrm{m}^{2}$ while bariatric surgery was performed for weight reduction at a mean BMI of $49.5 \mathrm{~kg} / \mathrm{m}^{2} \quad(p<0.0002)$ (Table 1$)$. The overall mean estimated body mass index loss calculated according to the recommendations for reporting weight loss [15] was 84.7\% (range, 32.5-156.7\%). Dietetically achieved weight reduction was hereby statistically significantly higher than the surgically induced reduction $(p<0.0001$; mean dietetic

Table 1 Details of analyzed patients' cohort

\begin{tabular}{lll}
\hline & Bariatric & Dietetic \\
\hline Number of patients $(n)$ & $62(59.6 \%)$ & $42(40.4 \%)$ \\
Age (years) & $40.3(23-64)$ & $40.1(19-63)$ \\
Male & $11(17.7 \%)$ & $6(14.3 \%)$ \\
Female & $51(82.3 \%)$ & $36(85.7 \%)$ \\
Smoker & $27(43.5 \%)$ & $20(47.6 \%)$ \\
Pre-bariatric BMI* $(\mathrm{kg} / \mathrm{m} 2)$ & 49.5 & 40.4 \\
EBMIL $(\%)^{* *}$ & $80.80 \%$ & $94.90 \%$ \\
BMI before body contouring $(\mathrm{kg} / \mathrm{m} 2)$ & 30.3 & 26.2 \\
Hernias*** & $21(33.9 \%)$ & $6(14.3 \%)$ \\
\hline
\end{tabular}

Cohort details, significant differences in characteristics ${ }^{*} p<0.1 ; * * p<0.01 ; * * * p<0.01$
EBMIL 94.90\% vs. mean EBMIL surgical 80.80\%) leading to a mean overall preoperative BMI of $29.7 \mathrm{~kg} / \mathrm{m}^{2}$ (Table 1).

Reconstructive body contouring therapy included in 75 patients $(72.1 \%)$ abdominoplasty and in 29 patients $(27.9 \%)$ belt lipectomy. The mean operative time was $3.5 \mathrm{~h}$. The mean total resection weight was $2,996 \mathrm{~g}$ per patient. The soft tissue resection weight during body contouring showed a significant negative correlation with EBMIL ( $r=-0.55, r^{2}=0.3, p<0.0001$, Fig. 2 ).

Intraoperative findings during body contouring surgery included hernias in 27 patients $(26 \%)$ which underwent intraoperative repair. Incisional hernias were found in 14, umbilical hernias were present in 8 and abdominal wall hernias in 5 patients. Unsurprisingly, patients following bariatric surgery showed a statistically significantly higher incidence of incisional hernias compared to patients with dietetic weight reduction $(p<0.01)$. The incidence of umbilical hernias showed no difference in the two weight reduction groups. The overall risk for hernia occurrence in patients following massive weight reduction was significantly elevated over 40 years of age $(p<0.05)$.

According to the defined criteria, the total complication rate following reconstructive body contouring surgery was $26.9 \%$. The most frequent complications were wound dehiscences that occurred in 21 patients $(20 \%)$ of patients and were treated predominantly conservatively. The second most frequent complication was seroma formation in 8 patients (7.7\%) (Fig. 3). Surgical revision was necessary in 14 patients (13.4\%). Despite routinely administered antithrombotic therapy, one patient developed a lethal pulmonary embolism 3 weeks following discharge from hospital. Complications were not dependent of age, gender, BMI max, or EBMIL. The BMI prior to body contouring surgery (BMI preop) had however a significant impact on the infection rate $(p<0.05)$, but was not statistically linked to other complications.

The total resection weight during the body contouring procedure influenced significantly the postoperative necrosis and wound dehiscence rate $(p<0.05)$.

Bariatric surgery patients presented with a significantly lower preoperative hemoglobin level than patients following dietetic weight reduction $(p<0.05)$. Low preoperative hemoglobin levels did not show a statistically significant increase in postoperative complication rates nor did the estimated intraoperative blood loss. The mean overall estimated intraoperative blood loss was $844 \mathrm{ml}$ and 22 patients received blood transfusions. A total of 47 patients (45.2\%) admitted to smoking but no statistically significant increase in their complication rates was observed.

The data collected in this study allowed-for future prospective investigations - by means of power analysis the assessment of the group sample sizes necessary to achieve $80 \%$ power to detect an odds ratio in the group proportions 
Fig. 1 Therapeutic approach leading to massive weight reduction prior to body contouring surgery

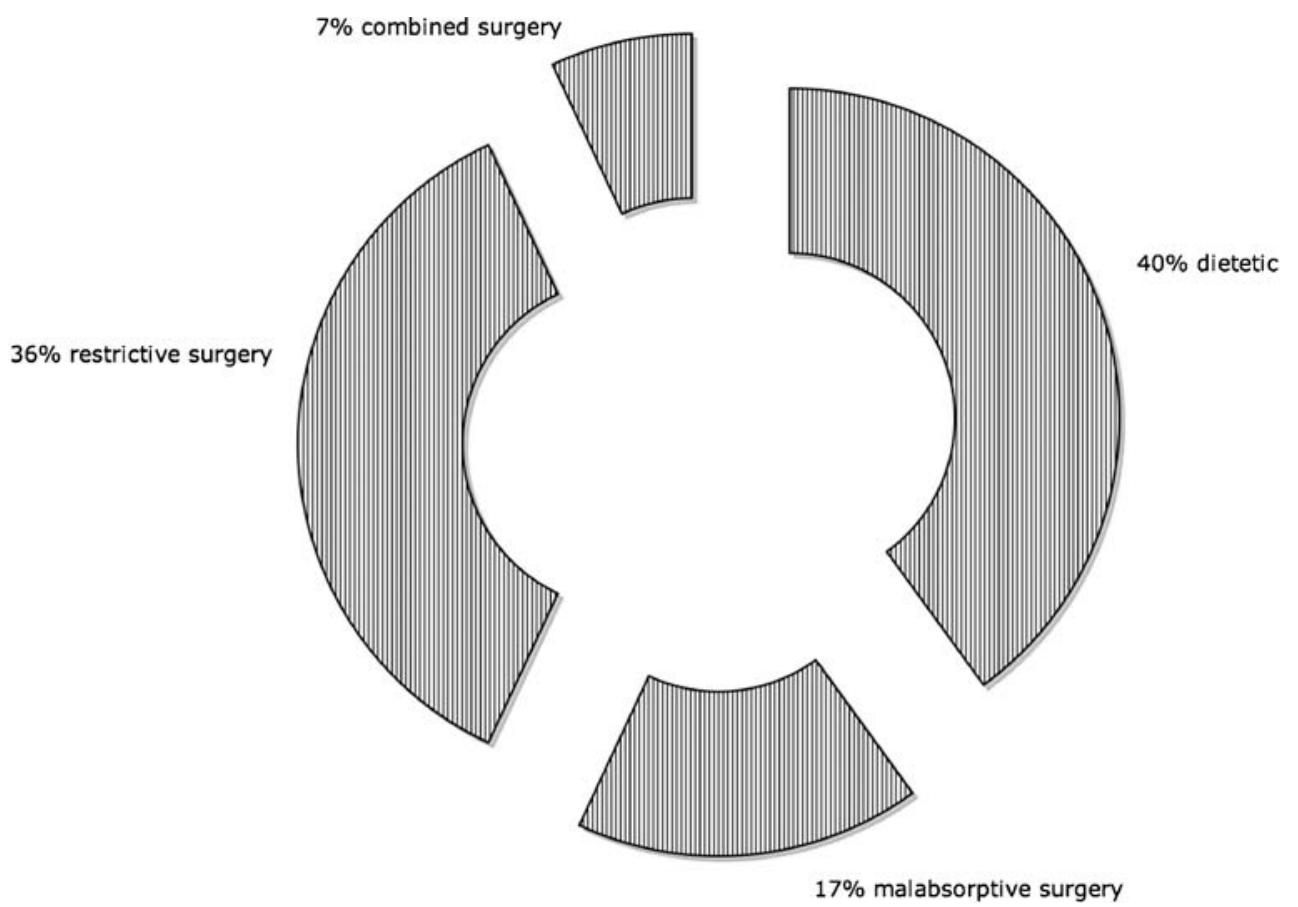

of 1.6892 targeting a significance level of 0.025 (since no previous direction was postulated). The calculation was performed using a two-sided Fischer's Exact test. The required total number of patients for prospective studies is estimated at $n=654$ (with $n=327$ per treatment group).

\section{Discussion}

Patients suffering from morbid obesity who successfully undergo massive weight loss are an inhomogenous group with various comorbidities, different success rates, and different preceding treatment methods. Recent studies

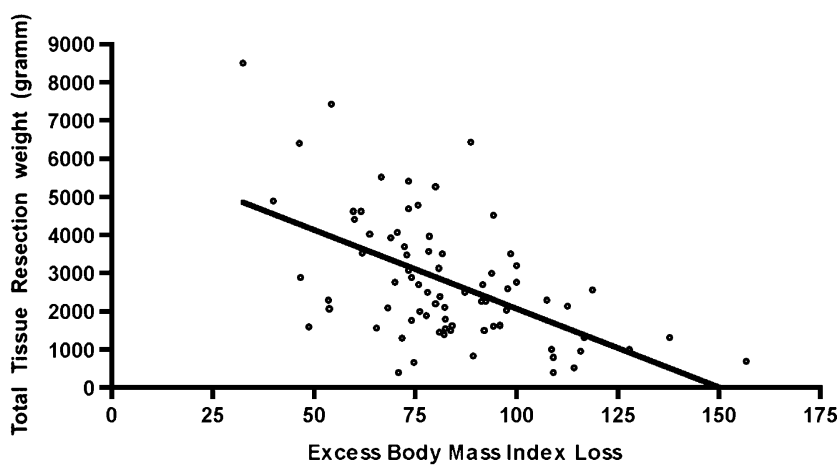

Fig. 2 Negative correlation of overall soft tissue resection weight during body contouring surgery and EBMIL. A higher achieved preoperative EBMIL leads to a lower necessary soft tissue resection weight compared the impact of the therapeutic approach on comorbidities and quality of life improvement [6, 17], but no comparative data was issued on the residual risks for subsequent reconstructive body contouring surgery following successful massive weight reduction. Common sequels of successful weight reduction remain stigmatizing in form of excess skin and soft tissues. These combined borderline conditions between medical and aesthetic limitations have to be addressed by reconstructive body contouring surgery in order to complete social and psychological reintegration following the long suffering of these patients [18].

Bariatric surgery is known to achieve successfully and rapidly massive weight reduction but may induce nutritional imbalance through malabsorption and intake restriction $[19,20]$. In comparison, dietetic weight reduction occurs slower with a postulated lower risk for nutritional imbalance but also with a lower success rate in terms of overall EBMIL [6]. The various therapeutic methods of weight reduction may therefore have different impact on subsequent wound healing, blood transfusion need, and postoperative complication rates following secondary body contouring surgery.

The presented retrospective study compared possible risk factors for complications in both dietetic and bariatric surgical patient groups at secondary body contouring procedures following massive weight loss and did not outline any differences in the risk analysis between the two groups. The selection criteria for body contouring surgery in the 104 patients were particularly strict in this previously morbidly obese population (BMI $\max \geq 35 \mathrm{~kg} / \mathrm{m}^{2}$ ). All patients had first to achieve a successful weight reduction 
Fig. 3 Detailed distribution of complications within the overall observed $26.9 \%$ complication rate


of EBMIL $\geq 30 \%$ irrespective of the preceding weight loss therapy and maintain a stable plateau phase for 12 months. These high requirements prior to body contouring surgery were based on preliminary observations from our institution [13] showing, similarly to other studies [10, 11, 13, 21], high complication rates exceeding $40 \%$ in patients with low EBMIL. As a consequence of these strict requirements for body contouring, the complication rate was successfully reduced to only $26.9 \%$ in this selected population.

The limitations of this study should be mentioned: firstly, the study design is retrospective and therefore detailed data acquisition is limited, secondly, patients' numbers are limited due to a single center database and literature meta-analysis is difficult in view of the different measures used in the past for weight loss quantification. Nevertheless, the collected data represents a basis for a power analysis for group sample sizes in a future prospective multicenter study design.

The current cohort compares well to patient populations in recent studies on this subject depicted in Table 2 Age, male gender, and smoking were found to be linked to higher complication rates by other authors [13, 21-24], but this observation was not paralleled by the present data.
Interestingly, despite an older patient population and similarly high percentage of smokers and the highest overall soft tissue resection weight, a low complication rate could be obtained in both dietetic and bariatric surgery patient groups of this study.

The possible impact of the preceding method of weight reduction on the subsequent body contouring surgery has not yet been sufficiently investigated. With the advent of EBMIL as an FDA approved and recognized measure for weight reduction [25], an appropriate mean for comparison of various weight reduction therapies and their individualrelated success has become available. This measure allows an improved inter-individual analysis of weight reduction between various therapies. The high EBMIL achieved both through dietetic therapy and bariatric surgery in this cohort underlines once more the strict preoperative patient selection. The EBMIL showed a negative correlation to the total tissue resection weight that was statistically linked to increased necrosis and wound dehiscence. This means the higher the weight loss, the less tissue weight has to be resected and the lower the risk for complications. Importantly, this finding was independent of the weight reduction method.

Table 2 Comparison of current data with observations from recent studies

\begin{tabular}{|c|c|c|c|c|c|c|c|c|c|c|c|c|}
\hline Author & $\begin{array}{l}\text { Patients } \\
\text { enrolled }\end{array}$ & Male & Female & $\begin{array}{l}\text { Mean } \\
\text { age }\end{array}$ & $\begin{array}{l}\text { Smoking } \\
\text { habits }\end{array}$ & EBMIL & $\begin{array}{l}\text { Maximal } \\
\text { BMI }\end{array}$ & $\begin{array}{l}\text { Pre-body } \\
\text { contouring } \\
\text { BMI }\end{array}$ & $\begin{array}{l}\text { Mean } \\
\text { weight } \\
\text { loss }(\mathrm{kg})\end{array}$ & $\begin{array}{l}\text { Tissue } \\
\text { resection } \\
\text { weight }(\mathrm{g})\end{array}$ & Hernia & $\begin{array}{l}\text { Complication } \\
\text { rate }(\%)\end{array}$ \\
\hline $\begin{array}{l}\text { Arthurs et al. } \\
\text { [11] }\end{array}$ & 126 & $\begin{array}{l}5 \\
(4 \%)\end{array}$ & $\begin{array}{l}121 \\
(96 \%)\end{array}$ & 42 & - & 82.6 & 48 & 29.0 & 53 & 1,200 & $\begin{array}{l}37 \\
(30 \%)\end{array}$ & 40 \\
\hline $\begin{array}{c}\text { Fraccalvieri } \\
\text { et al. [10] }\end{array}$ & 117 & $\begin{array}{l}16 \\
(13.7 \%)\end{array}$ & $\begin{array}{l}101 \\
(86.3 \%)\end{array}$ & 42 & $48 \%$ & 79.2 & 42.75 & 28.7 & 33.8 & 2,276 & $\begin{array}{l}13 \\
(11 \%)\end{array}$ & 50 \\
\hline $\begin{array}{l}\text { de Kerviler } \\
\text { et al. }\end{array}$ & 104 & $\begin{array}{l}17 \\
(16 \%)\end{array}$ & $\begin{array}{l}87 \\
(84 \%)\end{array}$ & 48 & $45.2 \%$ & 84.7 & 46.8 & 29.7 & 47.9 & 2,996 & $\begin{array}{l}28 \\
(27 \%)\end{array}$ & 26.9 \\
\hline
\end{tabular}


The toll of bariatric surgery has not gone unnoticed and is represented by the high rate of incisional hernias and the lower preoperative hemoglobin level when compared to the dietetic patient group. Although these observations parallel findings described by other authors [11, 22], they had no significant impact on the observed body contouring complication rates. According to Sugerman et al. [26] the incidence of incisional hernias following open gastric bypass operations was $25 \%$ and in a single group of superobese patients who underwent vertical banded gastroplasty it reached $50 \%$ [27]. Risk increases proportionally to BMI and with age ( $>40$ years) according to our observations. Although a future decrease of hernias can be expected with the advent of laparoscopic bariatric surgery, the reconstructive surgeon performing the body-contouring procedures has to be prepared to find and intraoperatively repair clinically silent hernias, and should be familiar with reconstructive techniques for the abdominal wall.

The achieved BMI prior to body-contouring surgery had only a significant impact on the observed infection rate, which parallels the findings of Arthurs et al. [11] who suggested BMI may become a selection criterion for bodycontouring surgery. The requested plateau phase in the mentioned study was however only 2 months. In view of the very low rate of complications achieved in the present study following both weight reduction modalities, a strictly defined minimal EBMIL of $30 \%$ or more in combination with a longer stable plateau phase may appear a more appropriate inclusion parameter. This is also in accordance with Larsen and Polat's request for a close-to-normal preop BMI prior to body contouring procedures [28]. When further compared to previous data from our own institution [13], the complications rate could be reduced by $50 \%$ with the above strict guidelines for patient selection, underlining this hypothesis. The advantages of this strict patient selection may have even outweighed certain risk factors like age and smoking habits.

\section{Conclusions}

Bariatric surgery does lead to increased incisional hernia rates and lower preoperative hemoglobin levels than dietetic weight loss but does not increase the risk of complications in secondary body contouring procedures. Strict selection criteria like a minimum EBMIL $\geq 30 \%$ and a long plateau phase of 12 months may be the key to a significant reduction of complications. A prospective multicenter analysis is planned.

Acknowledgement The authors would like to thank Dr. Franziska Schöni-Affolter, Institute of Evaluative Research, MEM-Center, University of Bern, for her specialist support in the statistical power analysis.

\section{References}

1. Kuczmarski RJ, Flegal KM, Campell SM, et al. Increasing prevalence of overweight among US adults. The national health and nutrition examination surveys, 1960 to 1991. Jama. 1994;272 (3):205-11.

2. Eichholzer M, Luthy J, Gutzwiller F. Epidemiology of overweight in Switzerland: results of the Swiss National Health Survey 199293. Schweiz Med Wochenschr. 1999;129(9):353-61.

3. Rickenbach M, Wietlisbach V, Beretta-Piccoli C, et al. Smoking, blood pressure and body weight in the Swiss population: MONICA study 1988-89. Schweiz Med Wochenschr Suppl. 1993;48:21-8.

4. NIH conference. Gastrointestinal surgery for severe obesity. Consensus Development Conference Panel. Ann Intern Med. 1991;115(12):956-61.

5. Fontaine KR, Redden DT, Wang C, et al. Years of life lost due to obesity. JAMA. 2003;289(2):187-93.

6. Karlsson J, Sjostrom L, Sullivan M. Swedish Obese Subjects (SOS)-an intervention study of obesity. Measuring psychosocial factors and health by means of short-form questionnaires. Results from a method study. J Clin Epidemiol. 1995;48(6):817-23.

7. Mathus-Vliegen EM, de Weerd S, de Wit LT. Health-related quality-of-life in patients with morbid obesity after gastric banding for surgically induced weight loss. Surgery. 2004;135 (5):489-97.

8. Fotopoulos L, Kehagias I, Kalfarentzos F. Dermolipectomies following weight loss after surgery for morbid obesity. Obes Surg. 2000;10(5):451-9.

9. Song AY, Rubin JP, Thomas V, et al. Body image and quality of life in post massive weight loss body contouring patients. Obesity (Silver Spring). 2006;14(9):1626-36.

10. Fraccalvieri M, Datta G, Bogetti P, et al. Abdominoplasty after weight loss in morbidly obese patients: a 4-year clinical experience. Obes Surg. 2007;17(10):1319-24.

11. Arthurs ZM, Cuadrado D, Sohn V, et al. Post-bariatric panniculectomy: pre-panniculectomy body mass index impacts the complication profile. Am J Surg. 2007;193(5):567-70; discussion 570.

12. Chaouat M, Levan P, Lalanne B, et al. Abdominal dermolipectomies: early postoperative complications and long-term unfavorable results. Plast Reconstr Surg. 2000;106(7):1614-8; discussion 1619-23.

13. Gmur RU, Banic A, Erni D. Is it safe to combine abdominoplasty with other dermolipectomy procedures to correct skin excess after weight loss? Ann Plast Surg. 2003;51(4):353-7.

14. van Uchelen JH, Werker PM, Kon M. Complications of abdominoplasty in 86 patients. Plast Reconstr Surg. 2001;107 (7):1869-73

15. Deitel M, Greenstein RJ. Recommendations for reporting weight loss. Obes Surg. 2003;13(2):159-60.

16. Pitanguy I, Gontijo de Amorim NF, Radwanski HN. Contour surgery in the patient with great weight loss. Aesthetic Plast Surg. 2000;24(6):406-11.

17. Shiri S, Gurevich T, Feintuch U, et al. Positive psychological impact of bariatric surgery. Obes Surg. 2007;17(5):663-8.

18. Scheufler O, Erdmann D. Current concepts and trends in postbariatric plastic surgery. Chirurg. 2007;78(4):316-25.

19. Faintuch J, Matsuda M, Cruz ME, et al. Severe protein-calorie malnutrition after bariatric procedures. Obes Surg. 2004;14 (2): $175-81$.

20. Davies DJ, Baxter JM, Baxter JN. Nutritional deficiencies after bariatric surgery. Obes Surg. 2007;17(9):1150-8.

21. Nemerofsky RB, Oliak DA, Capella JF. Body lift: an account of 200 consecutive cases in the massive weight loss patient. Plast Reconstr Surg. 2006;117(2):414-30. 
22. Stewart KJ, Stewart DA, Coghlan B, et al. Complications of 278 consecutive abdominoplasties. J Plast Reconstr Aesthet Surg. 2006;59(11):1152-5.

23. Shermak MA, Chang D, Magnuson TH, et al. An outcomes analysis of patients undergoing body contouring surgery after massive weight loss. Plast Reconstr Surg. 2006;118(4):102631.

24. Gravante G, Araco A, Sorge R, et al. Wound infections in post-bariatric patients undergoing body contouring abdominoplasty: the role of smoking. Obes Surg. 2007;17(10):132531 .
25. Deitel M, Gawdat K, Melissas J. Reporting weight loss 2007. Obes Surg. 2007;17(5):565-8.

26. Sugerman HJ, Kellum JM Jr, Reines HD, et al. Greater risk of incisional hernia with morbidly obese than steroid-dependent patients and low recurrence with prefascial polypropylene mesh. Am J Surg. 1996;171(1):80-4.

27. Arribas D, Elia M, Artigas C, et al. Incidence of incisional hernia following vertical banded gastroplasty. Hernia. 2004;8(2):135-7.

28. Larsen M, Polat F, Stook FP, et al. Satisfaction and complications in post-bariatric surgery abdominoplasty patients. Acta Chir Plast. 2007;49(4):95-8. 